See discussions, stats, and author profiles for this publication at: https://www.researchgate.net/publication/4238249

\title{
A fast and rigorous anisotropic smoothing method for DT-MRI
}

Conference Paper · May 2006

DOI: 10.1109/ISBI.2006.1624860 · Source: IEEE Xplore

\section{CITATIONS}

18

4 authors, including:

R. Deriche

National Institute for Research in Computer Science and Contro

443 PUBLICATIONS 22,460 CITATIONS

SEE PROFILE

Some of the authors of this publication are also working on these related projects:

High resolution whole brain diffusion MRI View project

Microstructure Imaging of Crossing (MIX) View project
READS

61

Juan Ruiz-Alzola

Universidad de Las Palmas de Gran Canaria

94 PUBLICATIONS 1,229 CITATIONS

SEE PROFILE 


\title{
A FAST AND RIGOROUS ANISOTROPIC SMOOTHING METHOD FOR DT-MRI
}

\author{
C.A. Castaño-Moraga ${ }^{1,2}$, C. Lenglet ${ }^{3}$, R. Deriche $^{3}$, J. Ruiz-Alzola ${ }^{1}$ \\ ${ }^{1}$ Centro de Tecnología Médica - Departamento de Señales y Comunicaciones \\ ${ }^{2}$ Departamento de Informática y Sistemas, Universidad de Las Palmas de Gran Canaria \\ Campus de Tafira s/n, 35017 Las Palmas de Gran Canaria, Spain \\ ${ }^{3}$ INRIA, Projet Odyssée, 2004 Route des Lucioles, 06902 Sophia-Antipolis, France
}

\begin{abstract}
Tensors are nowadays an increasing research domain in different areas, especially in image processing, motivated for example by DT-MRI (Diffusion Tensor Magnetic Resonance Imaging). In this paper, we exploit the theoretically wellfounded differential geometrical properties of the space of multivariate normal distributions, where it is possible to define a Riemannian metric and express statistics on the manifold of symmetric positive definite matrices. We focus on the contributions of these tools to the anisotropic filtering and regularization of tensor fields. We present promising results on synthetic and real DT-MRI data.
\end{abstract}

\section{INTRODUCTION}

DT-MRI (Diffusion Tensor Magnetic Resonance Imaging) is a relatively new medical imaging modality with many possible applications [2] and from which a great deal of research on tensors has stemmed. It can be estimated from a set of diffusion weighted images and provides a discrete three-dimensional dataset where each voxel contains a $3 \times 3$ symmetric positive definite matrix. These covariance matrices can be used to model the local anisotropic diffusion of water molecules by a Gaussian process. Working with these tensor fields may require some regularization to reduce the amount of noise arising, for instance, from the acquisition process.

Regularization and filtering schemes of tensor fields are widely studied in the literature, especially for DT-MRI. As an example, [11] proposed a PDE-based scheme based on a spectral decomposition. A complementary work is that presented in [5] and relying on constrained flows for matrix-valued functions. Another approach presented in [12] provides a generalization of anisotropic and nonlinear diffusion process to matrix-valued data. More recently, [6] proposes a scheme to recover the main direction of the tensors and use the resulting direction to regularize the eigenvalues by an anisotropic diffusion process. However, tensor eigenvalues tend to regularize faster than the asso- ciated eigenvectors. This phenomenon is known as the eigenvalue swelling effect for long regularization time, as shown in [11], while noise removal is not quite significant for short time regularization. Other works, such as [13], couple the regularization with the tensors estimation process from diffusion-weighted images.

In this paper, we use the mathematical framework presented in [8] and summarized in section 2 to take into account the particular geometry of the set of symmetric, positive definite matrices in the DT-MRI smoothing process. It is interesting to note that other recent works like [7] and [10] also addressed, from a Lie groups perspective, the definition of statistical quantities and filtering tools for tensor fields. We introduce an anisotropic filtering algorithm controlled by the magnitude of the spatial gradient of the tensor field in section 3. In section 4, qualitative and quantitative results obtained on noisy and synthetic datasets show that our method is favorably compared to a state-of-the-art approach [12]. We also present results obtained on different real datasets.

\section{DIFFUSION TENSOR PROCESSING TOOLS}

\subsection{Riemannian geometry of tensor space}

We consider the family of three-dimensional normal distributions with 0 -mean as the 6-dimensional parameter space of variances and covariances. We identify it with $S^{+}(3)$, the set of $3 \times 3$ real symmetric positive-definite matrices. A Riemannian metric can be introduced for $S^{+}(3)$ in terms of the Fisher information matrix [3]:

Theorem 2.1 The Riemannian metric for the space $S^{+}(3)$ of multivariate normal distributions with zero mean is given, $\forall \Sigma \in S^{+}(3)$ by:

$$
g_{i j}=g\left(E_{i}, E_{j}\right)=\left\langle E_{i}, E_{j}\right\rangle_{\Sigma}=\frac{1}{2} \operatorname{tr}\left(\Sigma^{-1} E_{i} \Sigma^{-1} E_{j}\right)
$$

where $\left\{E_{i}\right\} i, j=1, \ldots, 6$ denotes the basis of the tangent space $T_{\Sigma} S^{+}(3)=S_{\Sigma}(3)$ at $\Sigma \in S^{+}(3)$.

In other words, for any tangent vectors $A, B \in S(3)$, their inner product at $\Sigma$ is $\langle A, B\rangle_{\Sigma}=\frac{1}{2} \operatorname{tr}\left(\Sigma^{-1} A \Sigma^{-1} B\right)$. 
We can then define the length of a curve segment in $S^{+}(3)$ between two normal distributions parameterized by $\Sigma_{1}$ and $\Sigma_{2}$ and, hence, the geodesic distance [1].

Theorem 2.2 (S.T. Jensen, 1976) Consider the family of multivariate normal distributions with common mean vector but different covariance matrices. The geodesic distance between two members of the family with covariance matrices $\Sigma_{1}$ and $\Sigma_{2}$ is given by

$$
\mathcal{D}\left(\Sigma_{1}, \Sigma_{2}\right)=\sqrt{\frac{1}{2} \operatorname{tr}\left(\log ^{2}\left(\Sigma_{1}^{-1 / 2} \Sigma_{2} \Sigma_{1}^{-1 / 2}\right)\right)}
$$

Now, we can recall how the local average and spatial gradient of a diffusion tensor image can be computed.

\subsection{Weighted average of diffusion tensors}

An important practical application of theorem 2.2 is the ability to define statistics on $S^{+}(3)$, taking into account its special geometry. In this sense, the normal distribution parameterized by $\hat{\Sigma}^{w} \in S^{+}(3)$ and defined as the weighted intrinsic mean of $N$ distributions $\Sigma_{1}, \Sigma_{2}, \ldots, \Sigma_{N}$, achieves a minimum of the weighted sum of squared distances:

$$
\mu^{w}\left(\hat{\Sigma}^{w}, \Sigma_{1}, \Sigma_{2}, \ldots, \Sigma_{N}\right)=\frac{\sum_{k=1}^{N} \omega_{k} \mathcal{D}^{2}\left(\hat{\Sigma}^{w}, \Sigma_{k}\right)}{\sum_{k=1}^{N} \omega_{k}}
$$

A closed form expression cannot be obtained, but it is possible to derive a gradient descent algorithm for the computation of the intrinsic mean as shown in [8]. The associated numerical scheme is:

$$
\begin{gathered}
\hat{\Sigma}_{l+1}^{w}= \\
\hat{\Sigma}_{l}^{w \frac{1}{2}} \exp \left(-d t \frac{\hat{\Sigma}_{l}^{w \frac{1}{2}}\left(\sum_{k=1}^{N} \omega_{k} \log \left(\Sigma_{k}^{-1} \hat{\Sigma}_{l}^{w}\right)\right) \hat{\Sigma}_{l}^{w-\frac{1}{2}}}{\sum_{k=1}^{N} \omega_{k}}\right) \hat{\Sigma}_{l}^{w \frac{1}{2}}
\end{gathered}
$$

The algorithm simply starts from an initial guess $\hat{\Sigma}_{0}^{w}$ and follows the opposite of the gradient of the objective function (Eq. 2) along the geodesics of $S^{+}(3)$ to reach the minimum $\hat{\Sigma}_{l+1}^{w}$ in no more than $l=4$ or 5 iterations.

\subsection{Spatial gradient of diffusion tensor fields}

The magnitude of the spatial gradient of a tensor field can be estimated through the sum of squared geodesic distances between neighbors as follows:

$$
|\nabla \Sigma(x)|^{2} \simeq \sum_{k=1}^{3} \mathcal{D}^{2}\left(\Sigma(x), \Sigma\left(x \pm e_{k}\right)\right)
$$

where $\Sigma(x): \Omega \subset \mathbb{R}^{3} \mapsto S^{+}(3), \forall x \in \Omega$ is a tensor field defined in the spatial domain $\Omega$ and $\pm e_{k}$ are forward and backward elements of the canonical basis in $\mathbb{R}^{3}$ (see [4] for more details).

\section{DT-MRI ANISOTROPIC FILTERING}

The mathematical tools presented in the previous section allow us to develop an anisotropic smoothing algorithm for tensor fields regularization. In practice, we simply use the operator 3 to estimate local weighted averages. The anisotropic behavior is introduced by weighting each sample, within a local neighborhood, by a function that depends on the Riemannian gradient magnitude. This function is chosen so that, in homogeneous regions, the weights are constant and the tensors are isotropically averaged. On the contrary, when lying on an edge of the image, we would like that only samples on that boundary, and not those across, contribute to the local averaging. To achieve this goal and avoid mixing structures of the image, a possible choice for the weighting function is $w_{k}=\epsilon+|\nabla \Sigma(x)|^{2}$. A major advantage of this approach is that a straightforward $\mathrm{C}++$ implementation yields a quite computationally efficient algorithm since, to regularize a $50 \times 50 \times 50$ volume of $3 \times 3$ tensors, using a $3 \times 3 \times 3$ averaging neighborhood, we obtain an average processing time of 8 minutes on a $1.7 \mathrm{GHz}$ Pentium M CPU with $1 \mathrm{~Gb}$ of RAM. Moreover, it is easy to automatically detect the convergence of the gradient descent detailed in equation 3 by checking the evolution speed $\frac{\Sigma_{l}^{w}\left(\sum_{k=1}^{N} \omega_{k} \log \left(\Sigma_{k}^{-1} \hat{\Sigma}_{l}^{w}\right)\right)}{\sum_{k=1}^{N} \omega_{k}}$ and stopping whenever a given norm (Frobenius for instance) of this symmetric matrix has reached a certain threshold (1e-6 in practice). Hence not only do we ensure the convergence of the weighted mean but we also discard the need for a parameter such as the number of iterations.

\section{NUMERICAL EXPERIMENTS}

\subsection{Synthetic data}

In order to check the performance of our approach we generate a $32 \times 32 \times 32$ volume with the pattern shown on Fig. 1.a. Then, we generate the noisy version on Fig. 1.b. The noise follows a generalization of the Gaussian distribution for samples belonging to $S^{+}(3)$ using the algorithm proposed in [8] to generate a set of random positive definite tensors with the desired mean and covariance matrix. The noise model is consistent with the parametric model for noise in DT-MRI proposed in [9] where the authors proved that noise in DT-MRI data within a voxel follows a 6 -dimensional Normal distribution, assuming that the magnitude diffusion weighted images are Rician distributed. Fig. 1.c corresponds to the output of the anisotropic Riemannian filtering approach proposed in this paper, while Fig. 1.d is the output of a nonlinear matrixvalued diffusion scheme, as proposed in [12]. Differences arise when comparing these two images. First of all, noise is better removed with the Riemannian approach than with the nonlinear diffusion, where some misoriented tensors remain even after 536 iterations with a time step of 0.01 , which are the parameters that provide the optimal response. Furthermore, the well-known swelling effect, due to a faster reg- 


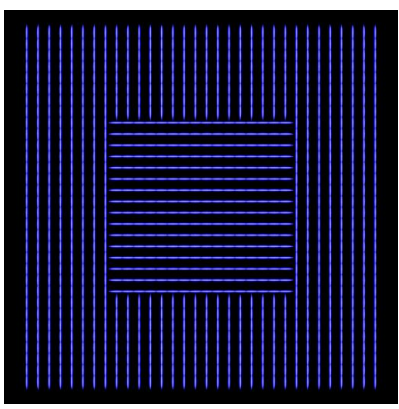

(a) Original Image

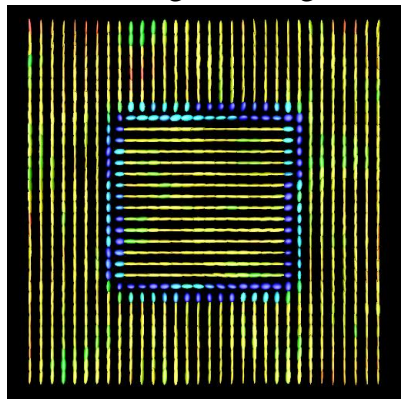

(c) Aniso. Riem. Smoothing

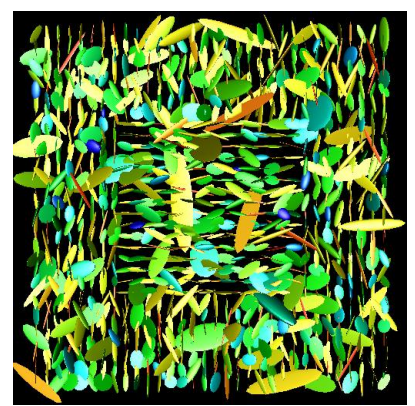

(b) Noisy Image

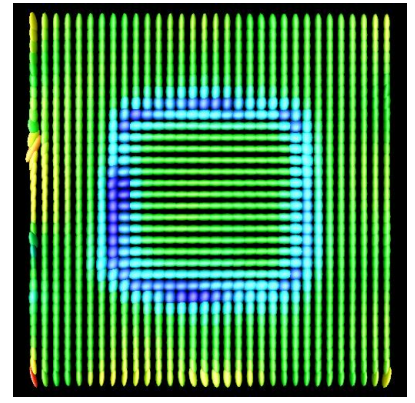

(d) Nonlin. Diff.
Fig. 1. Results on a $32 \times 32 \times 32$ synthetic cube.

ularization of the eigenvalues, is observed for the nonlinear diffusion, whereas it is not noticeable for our Riemannian approach. This can be observed by looking at the colors (blue: low FA and red: high FA) of the tensors: First, we point out that the original tensors are blue because they are all identical, thus have the same FA (0.77), and our visualization software assigns the color associated to the lowest value in that case. But most importantly, we can see that the regularized tensor field obtained with our approach is more anisotropic (tensors are yellow and FA is around 0.75) than that obtained with the nonlinear diffusion (tensors are green and FA is around 0.60). From a quantitative point of view, we measure the error between the original and regularized images by the geodesic distance between corresponding tensors. As shown in Table 1, the mean geodesic distance is much lower for the Riemannian anisotropic approach.

\subsection{Real DT-MRI data}

For experiments with real data, diffusion weighted images were acquired on a 3 Tesla scanner at the Centre IRMf de Marseille, France. We used 12 gradient directions and a bvalue of $1000 \mathrm{~s} / \mathrm{mm}^{2}$. Acquisitions were repeated 8 times for each direction to ensure a good signal-to-noise ratio. Voxel size was $2 \times 2 \times 2 \mathrm{~mm}^{3}$. Diffusion tensors shown on Figs. 2.a and 4.a were estimated by a robust gradient descent algorithm ensuring their symmetry and positive-definiteness, as presented in [8]. The idea of this method is to minimize a functional of the linearized Stejskal-Tanner equation by evolving an initial guess of the tensor on the manifold $S^{+}(3)$ with a numerical scheme similar to the one used for

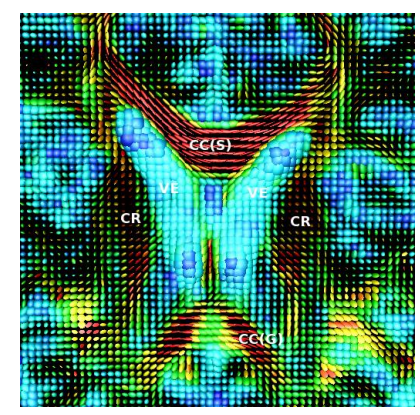

(a) Estimated Data

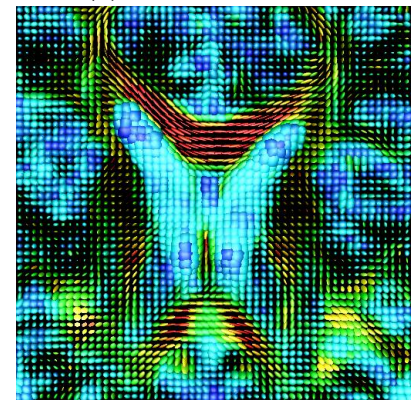

(c) Nonlin. Diff. $(\Delta t=0.001)$

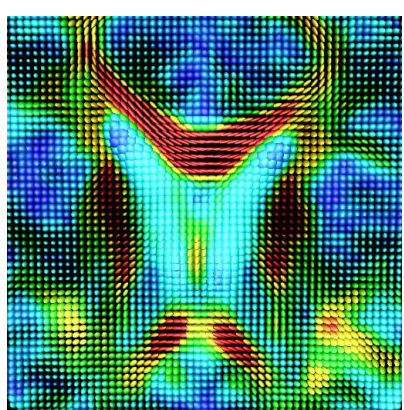

(b) Aniso. Riem. Smoothing

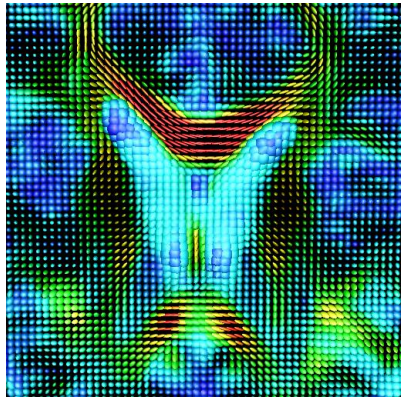

(d) Nonlin. Diff. $(\Delta t=0.01)$

Fig. 2. Results on DT-MRI data. a) Original DT-MRI data. b) Anisotropic Riemannian filtering. c) Nonlinear Diffusion - time step 0.001, 10 iter. d) Nonlinear Diffusion - time step 0.01, 10 iter. $($ Color code: Blue $=$ low FA and Red $=$ high FA)

the estimation of the average.

Fig. 2.b displays the regularized image using the anisotropic Riemannian smoothing, while bottom images are regularized using the nonlinear diffusion, both with 10 iterations, but different time steps. If we analyze the different structures on this axial slice, we can see that tensors orientation within the splenium and the genu of the corpus callosum $\mathrm{CC}(\mathrm{S})$ and $\mathrm{CC}(\mathrm{G})$ is more coherent with our Riemannian filtering scheme. Anisotropy in these areas is also better preserved than in the nonlinear diffusion case, which yields blurred areas most likely because of the properties of the Euclidean gradient. In addition, the ventricles VE, which are mainly homogeneous structures, are better regularized with our approach, as inhomogeneities do not disappear with the nonlinear diffusion. Finally, the corona radiata $\mathrm{CR}$ is well preserved with our approach while it is completely smoothed away from the image with a long diffusion time.

On Fig. 3 we show another DT-MRI volume where fiber orientation is color coded as follows: Red: Right-Left / Green: Anterior-Posterior / Blue: Inferior-Superior. Original data is shown on top of the image, while the filtered version is shown

\begin{tabular}{|c|c|c|c|c|}
\hline & Mean & Std. Dev. & Max & Min \\
\hline Aniso. Riem. & 0.2572 & 0.1313 & 1.3065 & 0.0300 \\
\hline Nonlin. Diff. & 0.8284 & 0.1209 & 3.2551 & 0.2630 \\
\hline
\end{tabular}

Table 1. Statistics on the error (geodesic distance) 


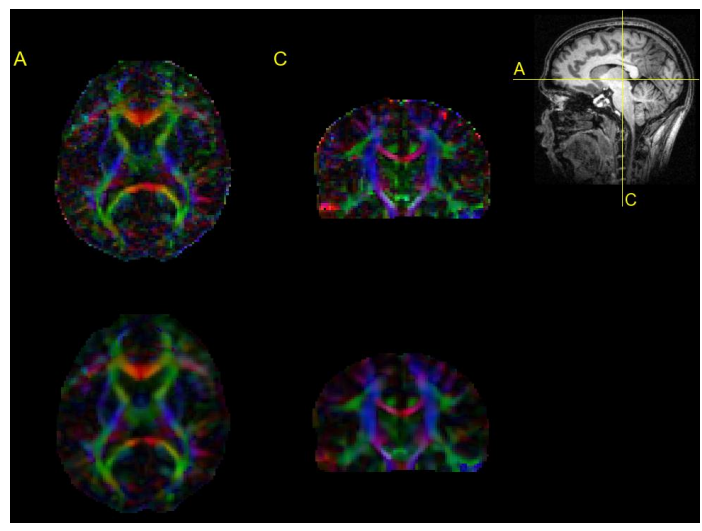

Fig. 3. Real data volume of a human brain.

at the bottom. A single slice from that volume is represented on Fig. 4, where we compare raw data (on the left) with the regularized version (on the right) using our approach.
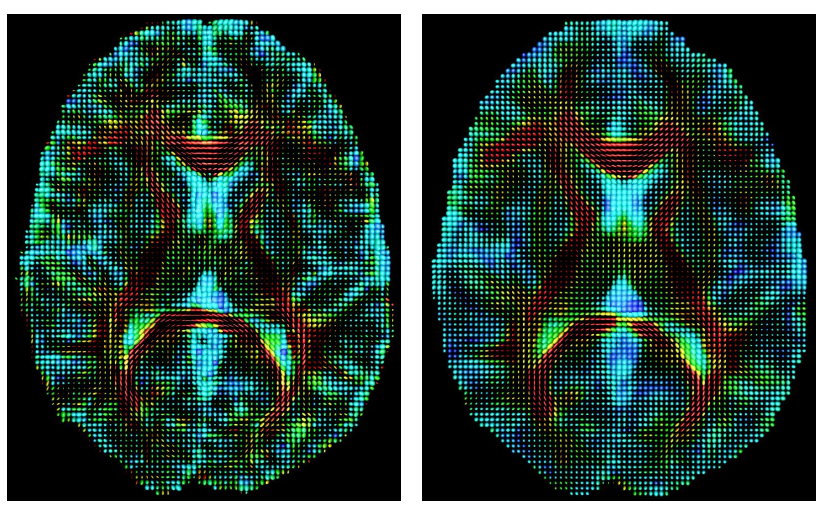

Fig. 4. [Left] Raw tensor field, [Right] Regularized tensor field

\section{CONCLUSION}

We have presented a novel differential geometrical approach for the anisotropic regularization of tensor fields, seen as fields of multivariate normal distributions. We focused on the properties of the space of multivariate normal distributions to introduce a Riemannian metric and notions such as the mean and spatial gradient which provide a well-founded framework to develop an anisotropic filtering algorithm for tensor data. The anisotropic behavior is introduced through the gradient magnitude, simply computed by using the geodesic distance between distributions. Our filtering scheme was compared to nonlinear diffusion of matrix-valued data to point out its added value and to show that it yields better results on synthetic and real DT-MRI data.

Acknowledgments: This research was partially supported by the European Commission (contract n. FP6-507609), ACI Obs-Cerv, the IMAVIS European project and the Région Provence-Alpes-Côte d'Azur. The authors would like to thank J.L. Anton, M. Roth and N. Wotawa for the human brain DTI dataset used in this paper.

\section{REFERENCES}

[1] C. Atkinson and A.F.S. Mitchell. Rao's distance measure. Sankhya: The Indian J. of Stats., (43):345-365, 1981.

[2] D. Le Bihan, J.F. Mangin, C. Poupon, C.A. Clark, S. Pappata, N. Molko and H. Chabriat. Diffusion tensor imaging: Concepts and applications. J. Magn. Reson. Imaging, (13):534-546, 2001.

[3] J. Burbea and C.R. Rao. Entropy differential metric, distance and divergence measures in probability spaces: A unified approach. Journal of Multivariate Analysis, (12):575-596, 1982.

[4] C.A. Castaño-Moraga, C. Lenglet, R. Deriche, and J. Ruiz-Alzola. A Riemannian approach to anisotropic filtering of tensor fields. Signal Processing, Special issue on Tensor Signal Processing, (to appear), 2006.

[5] C. Chefd'hotel, D. Tschumperlé, R. Deriche, and O. Faugeras. Constrained flows on matrix-valued functions: application to diffusion tensor regularization. In Proc. ECCV, 251-265, Prague, 2002.

[6] O. Coulon, D. Alexander and S. Arridge. Diffusion tensor magnetic resonance image regularisation. Medical Image Analysis, (8)-1:47-67, 2004.

[7] P. Fletcher and S. Joshi. Principal geodesic analysis on symmetric spaces: Statistics of diffusion tensors. In Proc. CVAMIA, 87-98, 2004.

[8] C. Lenglet, M. Rousson, R. Deriche, and O. Faugeras. Statistics on the manifold of multivariate normal distributions: Theory and application to Diffusion Tensor MRI. $J$. of Mathematical Imaging and Vision, (In press) 2006. Also available as INRIA Research Report 5242 (June 2004).

[9] S. Pajevic and P. Basser. Parametric and non-parametric statistical analysis of DT-MRI data. Journal of Magnetic Resonance, (106):1-14, 2003.

[10] X. Pennec, P. Fillard, and N. Ayache. A Riemannian framework for tensor computing. International Journal of Computer Vision, (65)-1:41-66, 2005.

[11] D. Tschumperlé and R. Deriche. Variational frameworks for DT-MRI estimation, regularization and visualization. In Proc. ICCV, 116-122, Nice, 2003.

[12] J. Weickert and T. Brox. Diffusion and regularization of vector- and matrix-valued images. Inverse Problems, Image Analysis and Medical Imaging. Contemporary Mathematics, (313):251-268, 2002.

[13] Z. Wang, B. Vemuri, Y. Chen, T. Mareci. A constrained variational principle for direct estimation and smoothing of the diffusion tensor field from complex DWI. IEEE Transactions on Medical Imaging, (23)-8:930-939, 2004. 